05

\title{
Влияние теплофизических свойств подложки анизотропного термоэлемента на величину термоэдс в нестационарном тепловом режиме
}

\author{
(C) П.А. Попов, С.В. Бобашев, Б.И. Резников, В.А. Сахаров
}

Физико-технический институт им. А.Ф. Иофффе РАН, Санкт-Петербург

E-mail: pavel.popov@mail.ioffe.ru

Поступило в Редакцию 5 июля 2016 г.

Рассчитаны поля температуры и электрического потенциала в модели теплового датчика на анизотропных термоэлементах при нестационарном тепловом режиме. Показано, что на начальном этапе нагрева влияние анизотропии теплопроводности материала термоэлемента мало, а распределение температуры близко к одномерному. При дальнейшем нагреве теплофизические свойства подложки существенно влияют на поле температуры и потенциала. Использование подложки с высокой температуропроводностью позволяет существенно упростить обработку результатов измерений, а также повысить электрический сигнал датчика.

DOI: 10.21883/PJTF.2017.07.44465.16396

Исследованию анизотропных термоэлементов, традиционно применяемых в качестве генераторов термоэдс или устройств охлаждения, посвящено большое количество публикаций $[1,2]$. В таких устройствах тепловой режим, как правило, считается известным, а предметом исследования являются особенности генерации термоэдс. В последнее время термоэлементы нашли применение в физических экспериментах в качестве чувствительных элементов тепловых датчиков. В этом случае ставится обратная задача - определение величины теплового воздействия по электрическому сигналу, полученному в эксперименте. Ее решение в широком диапазоне времен затруднено сложной зависимостью сигнала датчика от распределения температуры в анизотропном термоэлементе. В данной работе предлагается подход, основанный на зависимости распределения температуры в термоэлементе в силу 
анизотропии теплопроводности от теплофизических свойств подложки. В частности, он позволяет добиться близкого к одномерному профиля температуры, что существенно упрощает обработку результатов измерений датчиком такого типа.

Тепловой датчик представляет собой батарею последовательно соединенных термоэлементов из висмута длиной 1-10 $\mathrm{mm}$, закрепленную на подложке из слюды $[3,4]$. Он обладает малым временем отклика на тепловое воздействие, высокой чувствительностью и помехозащищенностью и успешно применяется в различных теплофизических экспериментах, в том числе на импульсных газодинамических установках в присутствии магнитных полей $~ 1 \mathrm{~T}[5-7]$.

Методика обработки результатов измерений датчиком на анизотропных термоэлементах зависит от теплового режима, в котором он находился в процессе регистрации теплового потока. Для режимов, близких к стационарному, тепловой поток $q$ может быть рассчитан поформуле $q=\frac{U}{S_{0} F}$, где $U-$ электрический сигнал датчика, $S_{0}-$ вольтваттная чувствительность, $F-$ площадь батареи термоэлементов [3]. Для нестационарного режима в настоящее время еще не предложено универсального метода расчета теплового потока по сигналу датчика, применимого для широкого диапазона времен. Причина состоит в том, что в силу аназотропии теплопроводности распределение температуры в термоэлементах двумерно, а зависимость сигнала датчика от теплового потока весьма сложна. Применение упомянутой выше формулы приводит к качественно неверным результатам [5,8]. Возможным решением является использование подложки с большой по сравнению с висмутом температуропроводностью, например медной $a \sim 10^{-4} \mathrm{~m}^{2} / \mathrm{s}$. Это позволяет добиться близкого к одномерному распределения температуры в термоэлементе [9], при котором справедлива формула Томсона [1] $\Delta \varphi_{T}(t)=\alpha_{12} \Delta T(t) l / h, \alpha_{12}$ - компонента тензора термоэдс, $\Delta T=T_{h}-T_{0}-$ разность температур между рабочей и тыльной поверхностью термоэлемента, $l / h-$ отношение его длины к толщине. Для этого случая был предложен удобный в экспериментальной практике метод расчета проходящего через датчик теплового потока по его электрическому сигналу [5].

В настоящей работе анализируется влияние теплофизических свойств подложки на применимость формулы Томсона при нестационарном тепловом режиме датчика. Будем использовать описанную ранее модель датчика [9]. Анизотропный термоэлемент прямоугольной

Письма в ЖТФ, 2017, том 43, вып. 7 
формы длиной $l$ и толщиной $h$ расположен на непроводящей подложке толщиной $s$. Через рабочую поверхность термоэлемента $y=h$ проходит тепловой поток $q_{h}$, боковые поверхности термоэлемента и подложки $x=0$ и $x=1$ теплоизолированы. На тыльной поверхности подложки $y=s$ осуществляется конвективный теплообмен $q_{s}=\alpha_{s}\left(T_{s}-T_{e}\right)$, где $\alpha_{s}$ - коэффициент теплоотдачи, $T_{s}$ и $T_{e}$ - температура подложки и окружающей среды. На всех поверхностях термоэлемента задается условие электроизоляции $j_{n}=0$. Физические свойства материалов датчика и подложки постоянны.

Далее рассматриваются два варианта модели теплового датчика. В первом (полная модель) учитывается анизотропия свойств материала термоэлемента, а распределение температуры $T(x, y)$ и потенциала $\varphi(x, y)$ находится из решения системы уравнений [2]

$$
C \rho \frac{\partial T}{\partial t}=\operatorname{div} \mathbf{q}, \quad \operatorname{div} \mathbf{j}=0,
$$

где $\mathbf{q}=-\lambda \nabla T, \mathbf{j}=-\sigma \nabla \varphi-\sigma \alpha \nabla T-$ плотность потока тепла и электрического тока; $T$ - температура; $\lambda, \sigma, \alpha-$ тензоры теплопроводности, электропроводности и термоэдс. Численное решение системы проводится в пакете COMSOL Multiphysics. Разность потенциалов $\Delta \varphi$ определяется между точками $A$ и $B$, расположенными на рабочей поверхности термоэлемента $y=h$. В [9] показано, что результаты расчета по данной модели хорошо совпадают с экспериментальными данными, полученными при стационарной калибровке соответствующих датчиков.

Во втором варианте (упрощенная модель) учитывается только анизотропия термоэдс, а теплопроводность материала термоэлемента изотропна. Распределение температуры $T=T(y)$ находится из решения уравнения теплопроводности, а разность потенциалов между точками А и В рассчитывается по формуле Томсона $\Delta \varphi_{T}=\alpha_{12} \Delta T(l / h)$, т. е. учитывается только поперечная термоэдс.

Рассмотрим типичные для датчиков термоэлементы длиной $l=2$, $5,10 \mathrm{~mm}$ и толщиной $h=0.2 \mathrm{~mm}(l / h=10,25,50)$, расположенные на подложках из слюды и меди. Моделируется нагрев постоянным тепловым потоком $q_{n}=100 \mathrm{~kW} / \mathrm{m}^{2}$ от начального состояния, когда $T=T_{e}$, до выхода на стационарный тепловой режим, когда $q_{s}=q_{h}$. Отличие разности потенциалов $\Delta \varphi$, рассчитанной по полной модели и

Письма в ЖТФ, 2017, том 43, вып. 7 

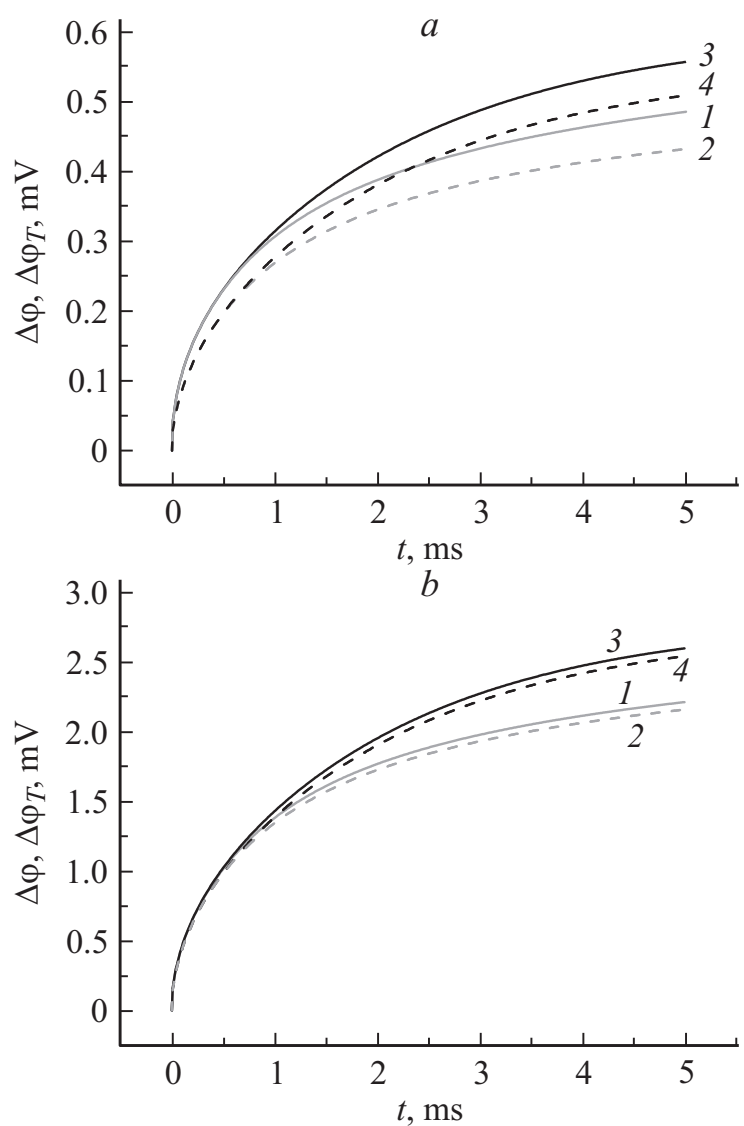

Рис. 1. Разность потенциалов на начальном этапе нагрева $(a, b)$ и выходе на стационарный режим $(c, d)$ для термоэлементов с $l / h=10(a, c)$ и $l / h=50(b, d)$. Кривые 1,2 соответствуют подложке из слюды, а 3,4 - из меди, при этом 1,3 рассчитаны по полной модели, а 2,4- по упрощенной.

рассматриваемой как электрический сигнал датчика, от $\Delta \varphi_{T}$, полученной по упрощенной модели, позволяет оценить ее применимость для расчета искомой разности температур $\Delta T=T_{h}-T_{0}$.

На рис. 1 показана разность потенциалов $\Delta \varphi$ и $\Delta \varphi_{T}$ для двух диапазонов времен. На начальном этапе нагрева температура тыльной

Письма в ЖТФ, 2017, том 43, вып. 7 

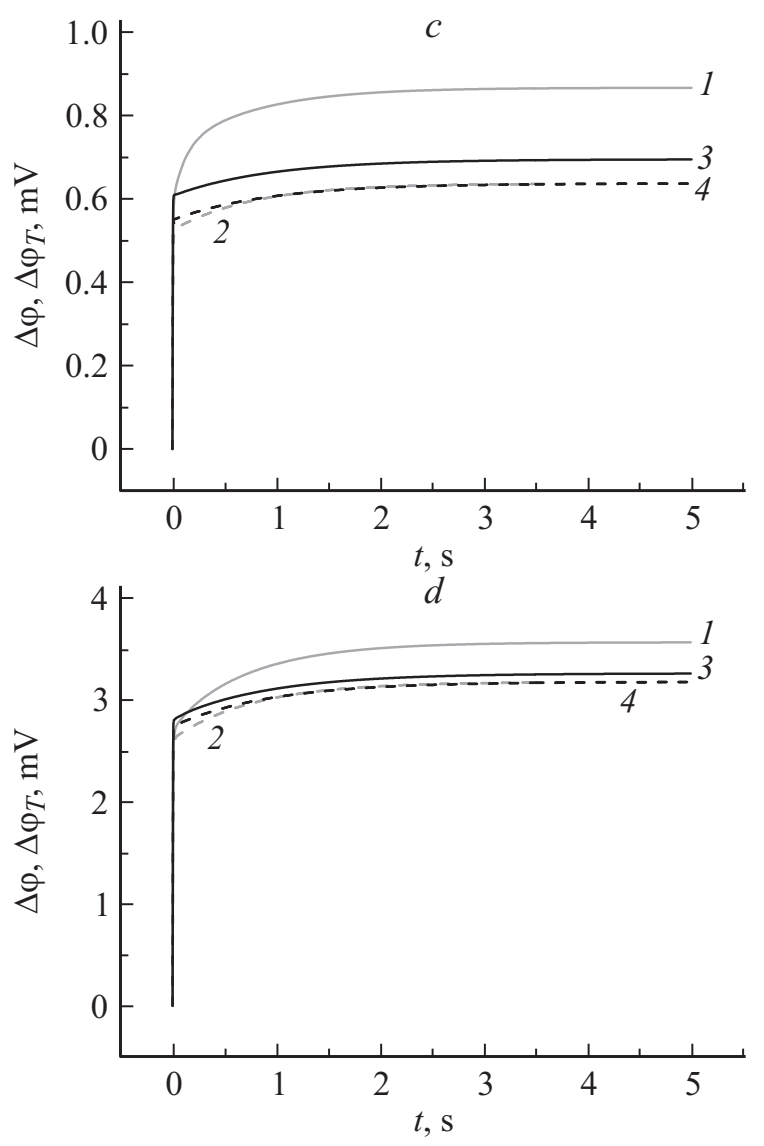

Рис. 1 (продолжение).

поверхности термоэлемента $T_{0}$ остается неизменной. Для рассматриваемых термоэлементов это время составляет $\approx 0.5 \mathrm{~ms}[10]$, в течение которого результаты расчета по обеим моделям близки (рис. $1, a, b)$. Это означает, что на данной стадии влияние свойств подложки и анизотропии теплопроводности материала термоэлемента мало, распределение температуры практически одномерно и определяющим является вклад только поперечной термоэдс. При дальнейшем нагреве теплофизиче-

Письма в ЖТФ, 2017, том 43, вып. 7 


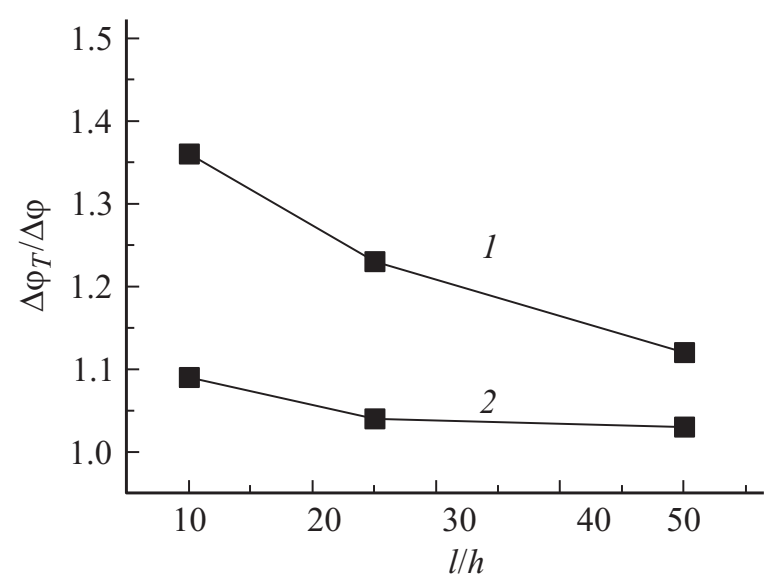

Рис. 2. Отношение термоэдс, рассчитанной по полной модели, к значению, полученному по упрощенной модели, для различных соотношений сторон $l / h$ термоэлемента, расположенного на подложке из слюды 1 и меди 2, при выходе на стационарный тепловой режим.

ские свойства материала подложки начинают влиять на распределение температуры в термоэлементе и соответственно термоэдс. В случае подложки из слюды поле температуры становится двумерным, и вклад продольной компоненты существенно возрастает, уменьшая результирующую термоэдс. В случае медной подложки распределение температуры остается близким к одномерному, при этом величина термоэдс выше для всех значений $l / h$. Для короткого термоэлемента наблюдается некоторое отличие $\Delta \varphi_{T}$ от $\Delta \varphi$ для обоих вариантов материала подложки, а в случае длинного термоэлемента кривые практически совпадают, что является следствием малого влияния анизотропии теплопроводности на данных временах.

При выходе на стационарный тепловой режим (рис. $1, c, d)$ сохраняется заметное влияние материала подложки на термоэдс. В случае подложки из слюды разность потенциалов, рассчитанная по полной (кривые 1,3 ) и упрощенной модели (кривые 2,4 ), существенно отличается, но для медной подложки это различие меньше. С увеличением длины термоэлемента это отличие уменьшается. Существенно, что в случае короткого термоэлемента и подложки из слюды зависимость

Письма в ЖТФ, 2017, том 43, вып. 7 
термоэдс немонотонна (кривая 1 на рис. 1,c). Это вызвано сопоставимым вкладом продольной и поперечной компонент которые имеют противоположные знаки. При расчете термоэдс по упрощенной модели зависимость термоэдс всегда монотонна (кривые 2 и 4), поскольку учитывается только поперечная компонента, пропорциональная разности температур $\Delta T$ между рабочей и тыльной поверхностью термоэлемента.

На рис. 2 показано отношение $\Delta \varphi_{T} / \Delta \varphi$ на достаточно больших временах, когда тепловой режим близок к стационарному. Видно, что в случае подложки из слюды использование упрощенной модели приводит к существенным погрешностям даже для достаточно длинных термоэлементов. Для медной подложки данный подход позволяет существенно повысить точность и при использовании коротких термоэлементов.

Расчеты показывают, что на начальном этапе нагрева упрощенная модель позволяет с высокой точностью определить по электрическому сигналу датчика искомую разность температур $\Delta T=T_{h}-T_{0}$. При измерении импульсных тепловых потоков длительностью $\sim 1 \mathrm{~ms}$ лучше использовать датчики с длинными термоэлементами $l / h \approx 50$, поскольку это увеличивает максимальное время применимости упрощенной модели. Использование подложки с большой температуропроводностью позволяет добиться близкого к одномерному распределения температуры в термоэлементе и применять упрощенную модель во всем диапазоне времен. Кроме того, это увеличивает выходной сигнал датчика при том же тепловом потоке. Следует отметить, что применимость упрощенной модели означает существование эффекта геометрической масштабируемости при расчете величины термоэдс. Это позволяет упростить процедуру предварительной калибровки, проведя ее для датчиков единичной площади, а затем использовать соответствующий масштабный коэффициент.

\section{Список литературы}

[1] Снарский А.А., Пальти А.М. и др. // ФТП. 1997. Т. 31. В. 11. С. 1281-1298.

[2] Анатычук Л.И. Термоэлектричество. Т. 2. Термоэлектрические преобразователи энергии. Киев: Институт термоэлектричества, 2003. $376 \mathrm{c}$.

[3] Сапожников С.3., Митяков В.Ю. и др. // ТВТ. 2004. Т. 42. В. 4. С 626-634.

[4] Mityakov A.V., Sapozhnikov S.Z. et al. // Sens. Actuators A: Physical. 2012. V. 176. P 1-9.

[5] Бобашев С.В., Менде Н.П. и др. // Письма в ЖТФ. 2009. Т. 35. В. 5. С. 36-42.

Письма в ЖТФ, 2017, том 43, вып. 7 
[6] Бобашев С.В., Головачев Ю.П. и др. // ЖТФ. 2009. Т. 79. В. 1. С. 36-44.

[7] Бобашев С.В., Менде Н.П. и др. // ЖТФ. 2010. Т. 80. В. 12. С. 51-56.

[8] Reznikov B.I., Sakharov V.A. et al. // Proceedings of the 7th International Workshop on Magneto-Plasma-Aerodynamics in Aerospace Applications. Moscow, 2007.

[9] Бобашев С.В., Попов П.А. и др. // Письма в ЖТФ. 2016. Т. 42. В. 9. С. 32-39.

[10] Резников Б.И., Сахаров В.А. и др. // Письма в ЖТФ. 2008. Т. 34. В. 5. C 28-33. 